\title{
A note about the ground state of the hydrogen molecule
}

\author{
A.V. Turbiner* and N.L. Guevarat \\ Instituto de Ciencias Nucleares, Universidad Nacional Autónoma de México, \\ Apartado Postal 70-543, 04510 México, D.F., Mexico
}

\begin{abstract}
A trial function is presented for the $H_{2}$ molecule which provides the most accurate (the lowest) Bohr-Oppenheimer ground state energy among few-parametric trial functions (with $\leq 14$ parameters). It includes the electronic correlation term in the form $\sim \exp \left(\gamma r_{12}\right)$ where $\gamma$ is a variational parameter.
\end{abstract}

*Electronic address: turbiner@nucleares.unam.mx

${ }^{\dagger}$ Electronic address: nicolais@nucleares.unam.mx 


\section{INTRODUCTION}

Hydrogen molecule $\mathrm{H}_{2}$ is among the most important chemical objects which appear in Nature. Since early days of quantum mechanics after pioneering paper by James and Coolidge 11] many studies of $H_{2}$ were carried out (see [2] and references therein). The paper [1] contained a clear indication that the interelectron correlation must be included explicitly. In general, the success of calculations and, in particular, a rate of convergence of a method used depends very much on a form of the correlation factor [3, 4, 5] (for a review, see [6], Section 2.2). In particular, recently, it was drawn a conclusion based on an analysis of many atomic and molecular systems that the best form of correlation factor is $\exp \left(\gamma r_{12}\right)$ comparing to linear or the Gaussian in $r_{12}$ factors [19]. No clear reason was given so far why it is so. A goal of this note is to present a simple, compact, easy-to-handle trial function which leads to the most accurate (the lowest) Bohr-Oppenheimer ground state energy among few-parametric trial functions ( $\leq 14$ parameters). The variational energy is calculated numerically using a specially designed computer code for multidimensional numerical integration with high accuracy. The trial function contains interelectron correlation in the form $\exp \left(\gamma r_{12}\right)$. It is worth mentioning that long time ago this dependence on $r_{12}$ appeared in the variational trial functions in studies of the $H_{2}$ molecule in a magnetic field [8] and, recently, of other two-electron molecular systems in a magnetic field [9, 10, 11]. A hint why namely this $r_{12}$-dependence leads to the fast convergent results will be given.

The Hamiltonian which describes the hydrogen molecule under the assumption that the protons are infinitely massive (the Born-Oppenheimer approximation of zero order) can be written as follows

$$
\mathcal{H}=\sum_{\ell=1}^{2} \hat{\mathbf{p}}_{\ell}^{2}-\sum_{\substack{\ell=1,2 \\ \kappa=A, B}} \frac{2}{r_{\ell, \kappa}}+\frac{2}{r_{12}}+\frac{2}{R},
$$

where $\hat{\mathbf{p}}_{\ell}=-i \nabla_{\ell}$ is the 3-vector of the momentum of the $\ell$ th electron, the index $\kappa$ runs over protons $A$ and $B, r_{\ell, \kappa}$ is the distance between $\ell$ th electron and $\kappa$ proton, $r_{12}$ is the interelectron distance, $R$ is the interproton distance. It is the established fact that the ground state of the $H_{2}$ molecule is ${ }^{1} \Sigma_{g}^{+}$, the spin-singlet state, symmetric under permutations of electron positions as well as proton positions. 


\section{VARIATIONAL METHOD}

The variational procedure is used as a method to explore the problem. The recipe of choosing the trial function is based on a physical relevance arguments (see, e.g. [12]). In practice, use of such trial functions implies the convergence of a special form of the perturbation theory where the variational energy is the sum of the first two terms. Let us remind the essentials of this perturbation theory (for details, see [12]). Let us assume that our original Hamiltonian has a form $\mathcal{H}=-\Delta+V$. As a first step we choose a trial function $\psi^{(t r i a l)}$ which is normalized to one. Then we find a potential for which our trial function $\psi^{(\text {trial })}$ is the exact eigenfunction $V_{\text {trial }}=\frac{\Delta \psi^{(\text {trial })}}{\psi^{(\text {trial })}}$ with the energy $E_{\text {trial }}=0$. In a pure formal way we can construct a Hamiltonian $\mathcal{H}_{\text {trial }}=-\Delta+V_{\text {trial }}$ such that $\mathcal{H}_{\text {trial }} \psi^{(\text {trial })}=0$. It can be easily shown that the variational energy

$$
E_{\text {var }}=<\psi^{(\text {trial })}|\mathcal{H}| \psi^{(\text {trial })}>
$$

is nothing but the first two terms in the perturbation theory where the unperturbed problem is given by $\mathcal{H}_{\text {trial }}$ and the perturbation is the deviation of the original potential $V$ from the trial potential $V_{\text {trial }}$, namely, $V_{\text {perturbation }}=V-V_{\text {trial }}$. Eventually, we arrive at the formula

$$
E_{\text {var }}=E_{\text {trial }}+E_{1}\left(V_{\text {perturbation }}\right)
$$

here $E_{1}\left(V_{\text {perturbation }}\right)=<\psi^{(\text {trial })}\left|V_{\text {perturbation }}\right| \psi^{(\text {trial })}>$ is the first energy correction in the perturbation theory, where unperturbed potential is $V_{\text {trial }}$. It is worth noting that if the trial function is the Hartree-Fock function the resulting perturbation theory is nothing but the Moeller-Plesset perturbation theory (see, e.g. [13], Section 15.18) [20].

One of the criteria of convergence of the perturbation theory in $V_{\text {perturbation }}=V-V_{\text {trial }}$ is a requirement that the ratio $\left|\frac{V_{\text {perturbation }}}{V}\right|$ should not grow when $r$ tends to infinity in any direction. If this ratio is bounded by a constant it should be less than one. In fact, it is a condition that the perturbation potential is subordinate with respect to the unperturbed potential. A value of this constant controls the rate of convergence - a smaller value of this constant leads to faster convergence [15]. Hence, the above condition gives a importance to the large-range behavior of the trial functions. In the physics language the above requirement means that the phenomenon of the Dyson's instability should not occur (for a discussion see [12]) [21]. Among three factors which are mentioned in literature (see [6]): the linear 
in $r_{12}$, exponential $\exp \left(\gamma r_{12}\right)$ and $\exp \left(-\alpha r_{12}^{2}\right)$, the only factor $\exp \left(\gamma r_{12}\right)$ fulfills the above requirement. It was demonstrated in [3] that a superposition of the Coulomb functions with exponentially correlated function $\exp \left(\gamma r_{12}\right)$ (see below, eq.(4)) leads to faster convergence than others. Perhaps, it is worth mentioning that for the case of Gaussian factor the abovedefined constant is equal to one exactly. In concrete, by following the above procedure and a requirement of the convergence of the perturbation theory we choose the trial function for the ground state in a form

$$
\psi^{(\text {trial })}=A_{1} \psi_{1}+A_{2} \psi_{2}+A_{3} \psi_{3}
$$

where

$$
\begin{aligned}
\psi_{1}= & \left(1+P_{12}\right)\left(1+P_{A B}\right) e^{-\alpha_{1} r_{1 A}-\alpha_{2} r_{1 B}-\alpha_{3} r_{2 A}-\alpha_{4} r_{2 B}+\gamma_{1} r_{12}}, \\
& \psi_{2}=\left(1+P_{12}\right) e^{-\alpha_{5}\left(r_{1 A}+r_{2 B}\right)-\alpha_{6}\left(r_{1 B}+r_{2 A}\right)+\gamma_{2} r_{12}}, \\
& \psi_{3}=\left(1+P_{12}\right) e^{-\alpha_{7}\left(r_{1 A}+r_{1 B}\right)-\alpha_{8}\left(r_{2 A}+r_{2 B}\right)+\gamma_{3} r_{12}},
\end{aligned}
$$

The $P_{12}$ is the operator which interchanges electrons $(1 \leftrightarrow 2)$ and $P_{A B}$ is the operator which interchanges the two nuclei $A \leftrightarrow B$. It is easy to check that the functions (5)-(6) are symmetric with respect to the interchange $A \leftrightarrow B$. The variational parameters consist of non-linear parameters $\alpha_{1-8}, \gamma_{1-3}$ which characterize (anti)screening of the Coulomb charges and linear parameters $A_{1-3}$. If the internuclear distance $R$ is taken into account the trial function (3) depends on 14 parameters [22]. It is worth mentioning that (5) is a degeneration of (44) when $\alpha_{1}=\alpha_{4}, \alpha_{2}=\alpha_{3}$ and (6) is another degeneration of (44) when $\alpha_{1}=\alpha_{2}, \alpha_{3}=\alpha_{4}$. In a certain way, the function (5) mimics the interaction of two hydrogen atoms $H+H$, while the function (6) mimics the interaction $\mathrm{H}_{2}^{+}+e$. Eventually, the function (4) can be treated as a non-linear interpolation between (5) and (6). Those functions look analogous to the Hund-Mulliken, Heitler-London and Guillemin-Zener functions, respectively.

Calculations were performed using the minimization package MINUIT from CERN-LIB. Multidimensional integration was carried out numerically using a "state-of-the-art" dynamical partitioning procedure: a domain of integration was divided into subdomains following an integrand profile, in particular, the domains with sharp changes of the integrand were separated out. Then each subdomain was integrated separately with controlled accuracy (for details, see e.g. [16]). A realization of the routine requires a lot of attention and care. During minimization process a partitioning was permanently controlled and adjusted. Numerical integration was done with a relative accuracy of $\sim 10^{-6}-10^{-7}$ by use of the adaptive 
D01FCF routine from NAG-LIB. Computations were performed on a dual DELL PC with two Xeon processors of $2.8 \mathrm{GHz}$ each.

\section{RESULTS}

Present results for the ground state of the $H_{2}$ molecule and their comparison with results of previous studies are displayed in Table I. The Bohr-Oppenheimer ground state energy obtained using the function (3)-(6) is the most accurate (the lowest) among those obtained with other trial functions with $\leq 14$ parameters. A reasonable agreement for expectation values is also observed, except for $\left\langle 3 z_{1}^{2}-r_{1}^{2}>\right.$ related to the quadrupole moment. To present authors it seems evident that this expectation value should be studied separately (see also [17]). It is not surprising that the obtained value of the cusp parameter in $r_{12}$ is equal to 0.4 unlike to the exact value 0.5 (see footnote [19]). Variational parameters of the trial function (3) are shown in Table II], It is worth emphasizing that a numerical calculations are very difficult and can easily lead to a loss of accuracy. In [3] a similar function (3) but with all three components of the form (4) containing 18 variational parameters was studied using variational Monte-Carlo technique. A comparison of our results with less parameters

with [3] (see Table I) indicates that we obtain a lower total energy of the order $5 \times 10^{-4} \mathrm{Ry}$.

\section{CONCLUSION}

We presented a simple and compact few-parametric trial function which provides the most accurate Bohr-Oppenheimer energy for $\mathrm{H}_{2}$ molecule among those based on few-parametric $(\leq 14)$ trial functions. Emerging five-dimensional integrals were effectively calculated using fast state-of-the-art integration routine which admits parallelization. The trial function (3) can be easily generalized by adding other physically-natural degenerations of (4) than (5), (6) . One of them appears when all $\alpha$-parameters in (44) are equal. It should be dominant in a domain of small interproton distances. It seems natural to assume that taking linear superpositions of the functions (4) we end up with fast convergent procedure (see [3]). The function (3) can be easily as modified for a study of spin-triplet states and as well as the states of the lowest energy with non-vanishing magnetic quantum numbers. A generalization to more-than-two electron systems is straightforward. 
TABLE I: Total energy $E_{T}$ in Ry and expectation values in a.u. of the hydrogen molecule $H_{2}$ for the ground state. $r_{1}, z_{1}$ are distances from 1 st electron to the mid-point between protons. Some data are rounded.

\begin{tabular}{|c|c|c|c|c|c|}
\hline$E_{T}(\mathrm{Ry})$ & $\left\langle r_{12}^{-1}>\right.$ & $<r_{1}^{2}>$ & $\left\langle\frac{\left(r_{1 A}+r_{1 B}\right)}{R}>\right.$ & $<3 z_{1}^{2}-r_{1}^{2}>$ & Refs. \\
\hline$-2.34697^{a}$ & & & & & {$[1]$} \\
$-2.34778^{b}$ & & & & & {$[18]$} \\
$-2.34787^{c}$ & & & & & {$[3]$} \\
$-2.348382^{d}$ & & 2.5347 & & 0.5227 & {$[17]$} \\
$-2.348393^{e}$ & 0.5874 & 2.5487 & 2.2133 & 0.4847 & present \\
$-2.34872^{f}$ & & 2.5426 & & 0.5142 & {$[17]$} \\
$-2.34888^{g}$ & 0.5874 & & 2.2127 & & {$[17]$} \\
$-2.34895^{h}$ & & & & & {$[2]$} \\
\hline
\end{tabular}

${ }^{a}$ from 1$]$ (the BO energy with 14 variational parameters)

${ }^{b}$ from [18] (based on use of $>200$ non-spherical Gaussian orbitals)

${ }^{c}$ from [3] ( $N=3$ exponentially correlated functions)

${ }^{d}$ from Table III [17] (the BO energy with 14 variational parameters)

${ }^{e}$ Present calculation (14 variational parameters)

$f_{\text {from Table III [17] (the BO energy with } 28 \text { variational parameters ) }}$

${ }^{g}$ from Table II [17] (the BO ground state energy with 40 variational parameters)

${ }^{h}$ from [2] (7034 James-Coolidge type terms, the record calculation at present, the number in Table is rounded))

\section{Acknowledgement}

This note is dedicated to the memory of Professor Koutecky with whom the first author had a privilege to talk extensively on several occasions in his very last years. It was very striking to see his openness and readiness to discuss new approaches in quantum chemistry. In fact, it was his encouragement that finally led to the present study.

The authors express their sincere gratitude to J.C. López Vieyra for the interest to the work and numerous useful discussions. The arguments and support by J. Cizek which convinced the present authors to publish presented results are highly appreciated.

This work was supported in part by PAPIIT grant IN121106 (Mexico). The first author is grateful to the University Program FENOMEC (UNAM, Mexico) for a support. 
TABLE II: Parameters of the trial function (3).

\begin{tabular}{|c|c|}
\hline $\mathrm{R}$ & 1.40053 \\
\hline$A_{1}$ & 1. \\
$\alpha_{1}$ & 0.720674986 \\
$\alpha_{2}$ & 0.521577488 \\
$\alpha_{3}$ & 0.130799743 \\
$\alpha_{4}$ & 1.30816746 \\
$\gamma_{1}$ & 0.0655006315 \\
\hline$A_{2}$ & -1.15105579 \\
$\alpha_{5}$ & 0.604583808 \\
$\alpha_{6}$ & 0.658402827 \\
$\gamma_{2}$ & -0.349101361 \\
\hline$A_{3}$ & 0.256342676 \\
$\alpha_{7}$ & 0.968330781 \\
$\alpha_{8}$ & 0.229153253 \\
$\gamma_{3}$ & -0.354509413 \\
\hline \hline
\end{tabular}

[1] H.M. James and A.S. Coolidge, J. Chem. Phys. 1, 825 (1933)

[2] J.S. Sims and S.A. Hangstrom, J. Chem. Phys. 124, 094101 (2006)

[3] D. Bressanini, M. Mella and G. Morosi, Chem. Phys. Lett. 240, 566 (1995)

[4] S. Ten-no, Chem. Phys. Lett. 398, 56 (2004)

[5] F.R. Manby et al. J. Chem. Phys. 124, 094103 (2006)

[6] W. Klopper et al. Int. Rev. Phys. Chem. 25, 427 (2006)

[7] F.B. Van Duijneveldt. 'Gaussian Basis Sets for the Atoms H-Ne for Use in Molecular Calculations', IBM Technical Report RJ 945

[8] A.V. Turbiner, Pis'ma ZhETF 38, 510(1983)

[9] A.V. Turbiner, N.L. Guevara and J.C. Lopez Vieyra, 'The $H_{3}^{+}$molecular ion in a magnetic field: linear parallel configuration' (physics/0606083)

[10] A.V. Turbiner and N.L. Guevara, 'The $\mathrm{He}_{2}^{++}$molecular ion can exist in a magnetic field', 
Phys.Rev. A74, 063419 (2006)

(astro-th/0610928)

[11] A.V. Turbiner, 'Molecular systems in a Strong Magnetic Field - how atomic-molecular physics in a strong magnetic field might looks like', Astrophysics and Space Science (2006) (in print)

[12] A.V. Turbiner, Usp. Fiz. Nauk. 144, 35 (1984)

Sov. Phys. - Uspekhi 27, 668 (1984) (English Translation)

[13] I.N. Levine, Quantum Chemistry, 5th Edition, Prentice-Hall, Upper Saddle River, New Jersey $07458(2000)$

[14] M.L. Leininger et al. J. Chem. Phys. 112, 9213 (2000)

[15] A.V. Turbiner, Yad. Fiz. 46, 204 (1987) Sov. Journ. of Nucl. Phys. 46, 125 (1987) (English Translation)

[16] A.V. Turbiner and J.C. López Vieyra, Phys. Repts. 424, 309 (2006)

[17] W. Kolos and C.C.J. Roothan, Rev. Mod. Phys. 32, 219 (1960)

[18] T. Detmer et al, Phys. Rev. A56, 1825 (1997)

[19] It is worth noting that any method based on the Gaussian in $r_{12}$ factor (or a superposition of the Gaussian functions as a factor) has an evident drawback - by construction the cusp parameter $\left.\frac{d \log \Psi}{d r_{12}}\right|_{r_{12}=0}$ is always zero (see, e.g., [3]). The fact, that those methods can lead to very accurate results for the energy, implies a relative unimportance of the short-range behavior in $r_{12}$ of the trial functions for getting the high-precision results in the calculation of the BO ground state energy. It also implies that these methods are doomed to a low accuracy of the expectation values for which a short-range behavior in $r_{12}$ of the wavefunction is important. This situation can be easily explored taking the H-atom as an example and applying a superposition of the Gaussian functions as a trial function [7]

[20] It is worth noting that the question about a convergence of the Moeller Plesset perturbation theory is not settled yet [14]

[21] It is worth noting that this procedure for a selection of the trial function was applied successfully to a study of one-electron molecular systems in a magnetic field leading to the highly accurate results. Many of these results are the most accurate at the moment (see [16]).

[22] Due to a freedom in normalization of the wave function one of the coefficients $A$ can be kept fixed; thus, in present calculation we put $A_{1}=1$ 